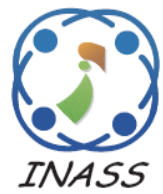

\title{
Performance of MIMO MC-CDMA for STBC Communication System Using OKHA Based Optimal Channel Estimation
}

\author{
Sreesudha Parnapalli ${ }^{1^{*}}$ \\ Barooru Lakshmi Malleswari ${ }^{2}$ \\ ${ }^{1}$ G. Narayanamma Institute of Technology and Science, Hyderabad, India \\ ${ }^{2}$ Sridevi Women's Engineering College, Hyderabad, India \\ Corresponding author's Email: sree.sudha38@gmail.com
}

\begin{abstract}
In the late years, MIMO MC-CDMA approach has been proposed in order to expand framework capacity over frequency-selective wireless channels. The key component of MIMO MC-CDMA is the capacity of abusing diversity jointly in time, frequency and in the space domain. In this work, we implement an efficient multi-user STBC MC-CDMA transmission System Based on Minimum Conditional BER Criterion and Oppositional krill herd algorithm (OKHA) assisted channel estimation. The multiuser MC-CDMA system using Alamouti's Space-Time Block coding for encoding the transmitting sequence. The estimation of Channel State Information (CSI) is optimally selected using OKHA. Normally, KHA is a natural-inspired metaheuristic algorithm which mimics the herding behavior of ocean krill individual. To improve the convergence speed and accuracy of the basic KHA algorithm, in this proposed work we combine KHA with oppositional based learning (OBL). The experimental results are conducted for the different algorithm based on BER, SER, and spectral efficiency. The simulation results show that the proposed algorithm is properly reducing the BER, SER and properly increase the spectral efficiency value compare to other techniques.
\end{abstract}

Keywords: MC-CDMA; STBC; Oppositional krill herd algorithm; Channel State Information; Minimum Conditional BER.

\section{Introduction}

Wireless communication is a rising field, which has seen enormous development in the most recent quite a long while. The development of video, voice and information correspondence over the Internet, and the similarly quick invasion of mobile telephony legitimize desires for mobile multimedia. Multicarrier CDMA frameworks have received impressive consideration with regards to wideband mobile communication systems as they consolidate high spectral productivity with an invulnerability to channel scattering [1]. The developing remote request alongside deficiencies in cell limit soon, different options, for example, visible light communication (VLC), have gotten genuine consideration as promising arrangements. Besides, the data transfer capacity impediments on the unlicensed radio recurrence (RF) range don't by any methods close the speed gap between RF remote and optical fiber systems [2]. The creating administrations require higher information rates from future cellular wireless communication.

To satisfy the necessities new advancements like Code Division Multiple Access and Orthogonal Frequency Division Multiplexing (OFDM) are few promising frameworks for the $4 \mathrm{G}$ correspondence guidelines. Unique codes are utilized recognize distinctive clients utilizing same recurrence band is the fundamental thought behind CDMA. The blend of OFDM and code-division multiple accesses get to (CDMA), known as MC-CDMA [3]. MC-CDMA is an exceptionally encouraging strategy for the future remote correspondence frameworks [4]. Multi-carrier code division multiple access get to (MC-CDMA), an innovation initially proposed in [5] and altogether outlined in [6], is a potential possibility for future remote frameworks. Be that as it may, MC-CDMA like all CDMA-based frameworks is restricted by the multiple access interference (MAI) incited by the loss of orthogonality among the clients after multipath 
propagation.

In this paper, we develop an efficient STBC MCCDMA transmission System Based on Minimum Conditional BER Criterion and Oppositional krill herd algorithm (OKHA) assisted channel estimation. Here, the channel matrix values are generated using OKHA because the channel matrix values basically affect the transmission signals. The OKHA algorithm is used to estimate the optimal channel matrix to decrease the MCBER. In this paper, to improve the efficiency of the system, oppositional based learning (OBL) is hybrid to the krill herd algorithm (KHA). In the experimentation, we analyze the performance of proposed work by varying number of transmitting and receiving antennas and compare our work with different approaches. This paper is organized as follows. In Section 2, we present the MC-CDMA system based some of the related works. In section 3, we have described a proposed STBC MC-CDMA system and section 4 explains the performance of proposed method and the simulation results are shown. Finally, we end the paper with brief conclusions in Section 5.

\section{Related Works}

Numerous works have been displayed in the literature for Communication system CDMA. Here, we audit a portion of the techniques presented. Phasouliotis et al. [7], proposed a chip level layered space -time (LST) collector design for coded downlink MIMO MC-CDMA frameworks. Here, they calculate the execution time and error rate of the work. But this method is very difficult. To overcome the difficulty Rasadurai et al. [8], proposed a turbo block code (TBC) procedure for enhancing the execution of a multi-carrier code division multiple access get to (MC-CDMA) framework as far as bit error rate (BER). In [9], de Figueiredo et al. explained a consistently turned finish reciprocal code (CRCCCs) in a code reuse plot alongside orthogonal space-time block codes (OSTBCs) and orthogonal frequency division multiplexing (OFDM). This system has low complexity but high BER. The Won et al [10], examined about the underlying synchronization in single antenna-assisted singleand multi-carrier Code Division Multiple Access (CDMA) and additionally Direct Sequence-Ultra Wide Band (DS-UWB) frameworks, with extraordinary accentuation on the DownLink (DL).

In [11], Pinhui et al. introduced a bland development of ZPCS sets in view of flawless successions and orthogonal frameworks. The parameters of our ZPCS sets are adaptable and are along these lines reasonable for various application situations. In [12], Shuai et al. proposed another framework engineering utilizing complete complementary $(\mathrm{CC})$ codes for a multicarrier MIMO CDMA framework to battle multipath blurring in remote channels. Liu et al. [13], proposed a fragmentary deferral strong beneficiary which is included a chip separated the corresponding exhibit by misusing the connection properties of CCC. Mottier et al. [14], proposed to perform BF on each subcarrier freely to permit any sort of detection algorithms at the versatile terminal. Likewise transmitting of Beamforming (BF) for SpaceDivision Multiple Access (SDMA) was considered in the downlink of remote cell frameworks in view of Multi-Carrier Code Division Multiple Access (MCCDMA). The above-mentioned methods are properly explained the CDMA-based signal transmission. To overcome the difficulty present in the above techniques in this paper we proposed an optimization approach based MIMO MC-CDMA for STBC communication System. Using optimization approach we can decrease the BER and reduce the loss. The used OKHA algorithm is recent algorithm it overcomes the difficulties present in the KHA algorithm. The complexity of the receiver is low compare to another approach.

\section{Proposed STBC MC-CDMA System for Channel Estimation}

The main objective of this paper is to the nearoptimum multiuser detector for STBC MC-CDMA transmission systems based on the Minimum Conditional Bit Error Rate (MCBER) and oppositional krill herd algorithm (OKHA). Here, at first, we generate the input signal consists of two symbols. Then, the input signals are mapped into two transmitting antennas which are encoded using STBC encoder. The encoder outputs are transmitted during two consecutive transmission periods, each one of duration equal to $T$. After that, the encoded signals are given to the IFFT. Finally, the RF-converted Multicarrier Spread Spectrum (MC-SS) signals are simultaneously transmitted by antenna 1 and antenna 2 , respectively. In this paper, we optimize the channel matrix using OKHA algorithm which gives the optimal result. Finally, we receive the signal without any loss and noise. The overall transmission process of proposed STBC MC-CDMA system is shown in figure 1 . 


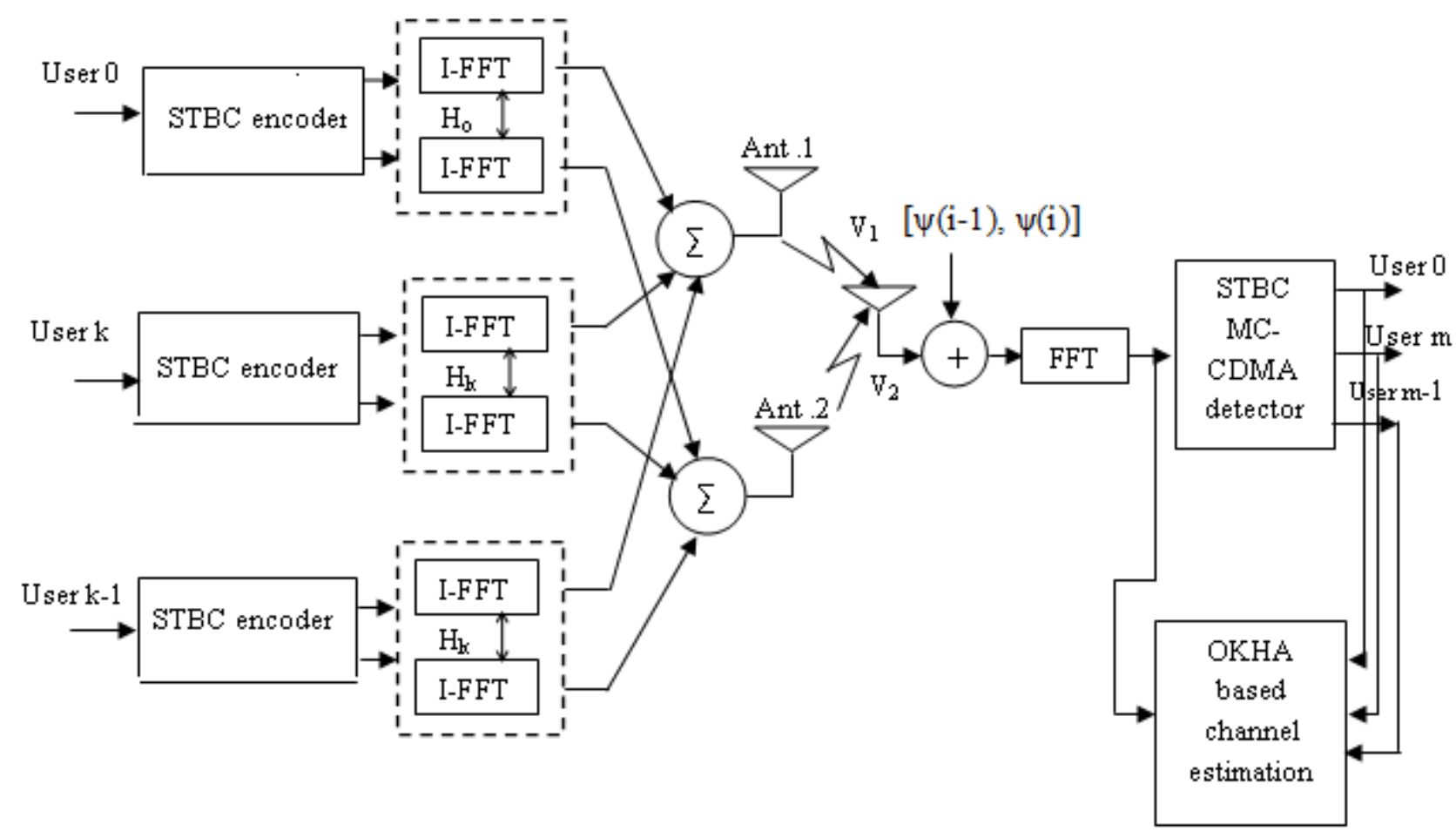

Figure.1 Overall architecture of STBC MC-CDMA system

\subsection{STBC MC-CDMA System Model}

In this work, we utilize synchronous multiuser MC-CDMA system based on STBC [15]. Here, we utilized transmitting $\mathrm{T}_{\mathrm{X}}$ and receiving antennas $\mathrm{R}_{\mathrm{X}}$. The block diagram of such a transmission system is shown in figure 1 . The figure shows the transmission between two transmitters and one receiving antennas. The STBC-CDMA system mainly focuses on two processes such as (i) transmitting signal and (ii) receiving signal. Here, in the transmitter side, we utilized the space-time block coding technique proposed by Alamouti in [3]. In figure 1, Two successive data symbols of the basic user $\mathrm{m}(m=0, \ldots$, $M-1)\left[p_{m}(i-1), p_{m}(i)\right]$ related to the basic $i^{\text {th }}$ modulation period $(i=1,3, \ldots)$ are mapped to two transmitting antennas according to the code matrix $\left[\phi_{m}(i)\right]$. The expression of the $2 \times 2$ orthogonal coding matrixes for the $m^{\text {th }}$ user at the generic $i^{\text {th }}$ signaling interval is given as follows:

$$
\phi_{m}(i)=\frac{1}{\sqrt{2}}\left[\begin{array}{ll}
p_{m}(i-1) & -p_{m}(i)^{\prime} \\
p_{m}(i) & p_{m}(i-1)
\end{array}\right]
$$

The superscript operator (') signifies the complex conjugate. The matrix of (1) signifies Alamouti's STBC block. The encoder outputs are transmitted through two successive transmission periods, i.e: $T 1$, $T 2$. Throughout the first transmission period $T 1$, the two QPSK symbols $p_{m}(i-1)$ and $p_{m}(i)$ are sent to two separate I-FFT based multicarrier spreading blocks using a unique Hadamard-Walsh sequence $c_{k} \hat{=}\left\{c_{k}\right.$ (n), $n=0, \ldots, N-1\}$ being $N$ the length of the I-FFT. Finally, the RF-converted multicarrier spread spectrum (MC-SS) signals are instantaneously transmitted by antenna 1 and antenna 2 , respectively.

In a similar manner, throughout the successive transmission period $T 2$, the symbol- $p_{m}(i)$ is transmitted by antenna 1 and the symbol- $p_{m}(i-1)$ is transmitted by antenna 2 , respectively. In order to make the notation more complex in the multiuser case, we define two vectors of symbols $P^{l} \hat{=}\left[p_{0}{ }^{l}, p_{l}{ }^{l}, \ldots\right.$, $\left.p^{l}{ }_{m-1}\right]^{T}$ and $P^{2} \hat{=}\left[p_{0}^{2}, p_{l}^{2}, \ldots, p_{m-1}^{2}\right]^{T}$. Moreover, we define the orthogonal code matrix $C$ as:

$$
C=\left[\begin{array}{cccc}
c_{0}(0) & c_{1}(0) & \ldots & c_{m-1}(0) \\
c_{0}(1) & c_{1}(1) & \ldots & c_{m-1}(1) \\
& \cdot & & \\
& \cdot & & \\
c_{0}(k-1) & c_{1}(k-1) & \ldots & c_{m-1}(k-1)
\end{array}\right]
$$

The received signal samples assimilated at two consecutive symbol periods after the FFT-based coherent de-multiplexing can be expressed as follows:

$$
\begin{array}{r}
R(i-1)=V_{1} C P(i-1)+V_{2} C P(i)+\Psi(i-1) \\
R(i)=-V_{1} C P(i)+V_{2} C P(i-1)+\Psi(i)
\end{array}
$$

Where, $R(i-1)$ and $R(i)$ and are $N \times 1$ vectors, $\psi(j)=\left[\psi_{0}(j), \psi_{1}(j), \ldots \ldots \ldots, \psi_{N-1}(j)\right]^{T}$ (with $\left.j \epsilon\{(i-1), i\}\right)$ is the additive white Gaussian noise (AWGN) vector (all vector mechanism are independent and 
identically scattered with zero mean and variance $\sigma^{2}$ ) and $V_{a n t}=\operatorname{diag}\left\{v_{0}{ }^{a n t}, h v_{1}^{a n t}, \ldots . ., h v_{N-1}{ }^{a n t}\right\}$ with ant $\epsilon\{1,2\}$ is a $N \times N$ diagonal channel matrix, being $v_{n}{ }^{a n t}$ the complex channel coefficient related to subcarrier and to the transmit antenna ant. We sensibly imagine that fading the coherence time is much greater than the symbol period.

\subsection{Proposed Optimal Channel Estimation System}

The objective of this section is to select the optimal channel metrics using Oppositional Krill Herd Algorithm (OKHA). Basically, KH algorithm is on the basis of simulating the herding behavior of krill individuals by a Lagrangian model and crossover. KHA pretends the herding behavior of krill swarms in response to precise biological and ecological procedures to resolve multi-dimensional, linear and nonlinear issues with considerable efficacy. In this optimization algorithm, the objective performance for the krill movement is supposed to be an amalgamation of the least distances of the position of the food and the uppermost density of the herd. The KHA repeat the application of the three movements and trails the search directions that augment the objective function value. To hasten the convergence speed and to recover the simulation solutions of this paper, opposition-based learning (OBL) is also combined in the basic KHA method. The step by step process of proposed OKHA algorithm based optimized Channel matrix selection procedure is enlightened below;

\section{Step 1: Solution Encoding}

Solution encoding is an important step for the optimization algorithm. In a realistic receiver, the channel matrices $V_{l}$ and $V_{2}$ are not deterministically known, but they should be replaced by their estimates $V^{\prime}{ }_{1}$ and $V^{\prime}{ }_{2}$. Here, at first, we randomly assign the value of channel matrices $V_{1}$ and $V_{2}$ based the transmitted input signal.

\section{Step 2: Opposite Solution Generation}

Let $p=(p 1, p 2, \ldots, p d)$ be a point in d-dimensional space, where $p_{m} \in\left[u_{m}, v_{m}\right]$ and $m=\{1,2, \ldots, d, \ldots, n\}$. The opposite point is defined by Eq. (5).

$$
p_{m}^{\prime}=u_{m}+v_{m}-p_{m}
$$

\section{Step 3: Fitness Calculation}

In this section, we have to minimize the objective function which is given in Eq. (6). The fitness function is designed based on the channel matrices transmitting signals. In this paper, we adopted the
MMSE channel estimate targeted to MIMO STBC system considers in [17].

$$
\begin{aligned}
& O\left(\hat{V}_{1}, \hat{V}_{2}\right)=\left\|R(i-1)-\hat{V}_{1} C P(i-1)-\hat{V}_{2} C P(i)\right\|^{2}+ \\
& \left\|R(i)+\hat{V}_{1} C P^{\prime}(i)-\hat{V}_{2} C P^{\prime}(i-1)\right\|^{2}
\end{aligned}
$$

\section{Step 3: Movement Induced by Other Krill Individuals}

In this procedure, the krill individuals try to sustain a high density though the velocity of each individual is prejudiced using the movement of the others. The direction of motion persuaded $\left(\Psi_{p}\right)$ is, roughly, assessed by the three effects, namely, $(a)$ local effect, $(b)$ target effect and $(c)$ repulsive effect. For a krill individual $\mathrm{p}$, this motion may be framed as;

$$
\begin{gathered}
W_{p}^{\text {new }}=\Psi_{P} W_{p}^{\max }+u_{w} W_{p}^{\text {old }} \\
\left(\Psi_{p}\right)=\sum_{q=1}^{n_{S}}\left[\frac{v_{p}-v_{q}}{v_{w}-v_{b}} \times \frac{u_{p}-u_{q}}{\left|u_{p}-u_{q}\right|+\operatorname{rand}(0,1)}\right]+X_{p}^{\text {best }}(8) \\
2\left[\operatorname{rand}(0,1)+\frac{z}{z_{\max }}\right] v_{p}^{\text {best }}
\end{gathered}
$$

Where, $W_{p}{ }^{\max }$ is the maximum induced motion, $u_{w}$ is the inertia weight of the motion encouraged in the range $[0,1], W_{p}^{\text {old }}$ is the preceding induced motion of the $p^{\text {th }}$ krill individuals, $u_{w}$ and $v_{b}$ are the worst and best position amongst all the krill individuals of the population, correspondingly. $v_{p}$ and $v_{q}$ are the fitness values of the $p^{\text {th }}$ and the $q^{\text {th }}$ individual correspondingly, $n_{S}$ is the number of krill individuals other than the specific krill, $z$ and $z_{\max }$ are corresponding, the number of current iteration and a maximum number of iterations and $u$ signifies the related positions. For the determination of the distance between the individual krill and the neighbor's a parameter named as sensing distance $\left(D_{s}\right)$ is used. It may be formulated by Eq. (9).

$$
D_{s}=\frac{1}{5 N_{p}} \sum_{k=1}^{N_{p}}\left|u_{p}-u_{k}\right|
$$

$D_{s}$ is the total number of the krill individual and $u_{p}, \quad u_{k}$ are the position of the pth, $k^{\text {th }}$ krill correspondingly. It is renowned now that when the distance within the two individual krill has lesser value than the sensing distance, then they are preserved as neighbors.

\section{Step 4: Calculate Foraging Activity}

Foraging activity is based upon two foremost factors. First are the current food location and the second is the data about the previous food location. 
The foraging velocity may be articulated for the $p^{\text {th }}$ krill individual by Eq. (10).

$$
\left.\begin{array}{l}
W_{F P}^{n e w}= \\
2 \times\left(1-\frac{Z}{Z_{\max }}\right) \times v_{p} \times \frac{\sum_{k=1}^{n s} \frac{u_{k}}{v_{k}}}{\sum_{k-1}^{n s} \frac{1}{v_{q}}} \\
+u_{w f} \times W_{F P}^{\text {old }}
\end{array}\right]
$$

Where, $u_{w f}$ is the inertia weight of the foraging motion, $W_{F P}{ }^{\text {new }}$ and $W_{F P}$ old are the foraging motions of the new and the old $p^{\text {th }}$ krill respectively.

\section{Step 5: Calculate Random Diffusion}

The random diffusion process of the krill individual is, mainly considered to augment the population diversity. It may be expressed as follows;

$$
W_{D P}^{n e w}=\alpha \times W_{D}^{\max }
$$

Where, $W_{D}{ }^{\max }$ are the maximum diffusion speed and $\alpha$ is the random directional vector lies between [$1,1]$.

\section{Step 6: Position Update}

In this procedure, the individual krill changes its current positions and transfers to better positions on the basis of induction motion, foraging motion and random diffusion motion. Rendering to the three above deliberated motions, the updated position of the $p^{\text {th }}$ krill individual may be articulated by Eq. (12).

$$
\begin{aligned}
& u_{p}^{n e w^{\prime}}=u_{p}^{n e w}+\left(W_{P}^{n e w}+W_{F P}^{n e w}+W_{D P}^{n e w}\right) \times \\
& P_{C} \sum_{q=1}^{n d}\left(U_{q}-L_{q}\right)
\end{aligned}
$$

Where $n_{d}$ is the entire number of variables, $U_{q}$ and $L q$ are the upper and the lower limits of the $q^{\text {th }}$ variables $\left(q=1,2, \ldots, n_{d}\right)$, correspondingly and PC is the position constant number within $[0,2]$. In order to progress the enactment of the optimization issue and to accelerate the convergence property, the crossover and also the mutation procedure of GA algorithm is assimilated with KHA.

\section{Step 7: Crossover Operation}

Crossover procedure is, principally, controlled using a parameter, termed as crossover probability (CR). In order to update the position of own, each krill individual interacts with others. In this procedure, the $q^{\text {th }}$ component of the $p^{\text {th }}$ krill may be expressed by Eq. (13).

$$
u_{p, q}= \begin{cases}u_{k, q} & \text { if rand } \leq C R \\ u_{p, q} & \text { if rand }<C R\end{cases}
$$

In this proposed work, we utilize the crossover probability rate is $0.2 v_{p}^{\text {best }}$.

\section{Step 8: Mutation Operation}

Mutation process is, mostly, controlled by a parameter termed as mutation probability (MR). This process may be formulated by Eq. (14).

$$
u_{p, q}=u_{b e s t, q}+\gamma\left(u_{m, q}-u_{n, q}\right)
$$

Where, $u_{\text {best }, q}$ is the worldwide best vector, $u_{m, q}$ and $u_{n, q}$ are the two arbitrarily selected vectors and c is a scalar number within 0 and 1 . The adapted value of $u_{p, q}$ may be designed by using Eq. (15).

$$
u_{p, q}^{\bmod }= \begin{cases}u_{p, q}^{n e w} & \text { if rand } \leq M R \\ u_{p, q} & \text { if rand }>M R\end{cases}
$$

\section{Step 9: Termination Criteria}

The algorithm discontinues its execution only if a maximum number of iterations is achieved and the solution which is holding the best fitness value is selected and it is specified as best channel matrix. Once the best fitness is attained by means of OKHA algorithm, selected solution is allocated for communication. The overall process of the OKHA based channel estimation is shown in figure 2 . 


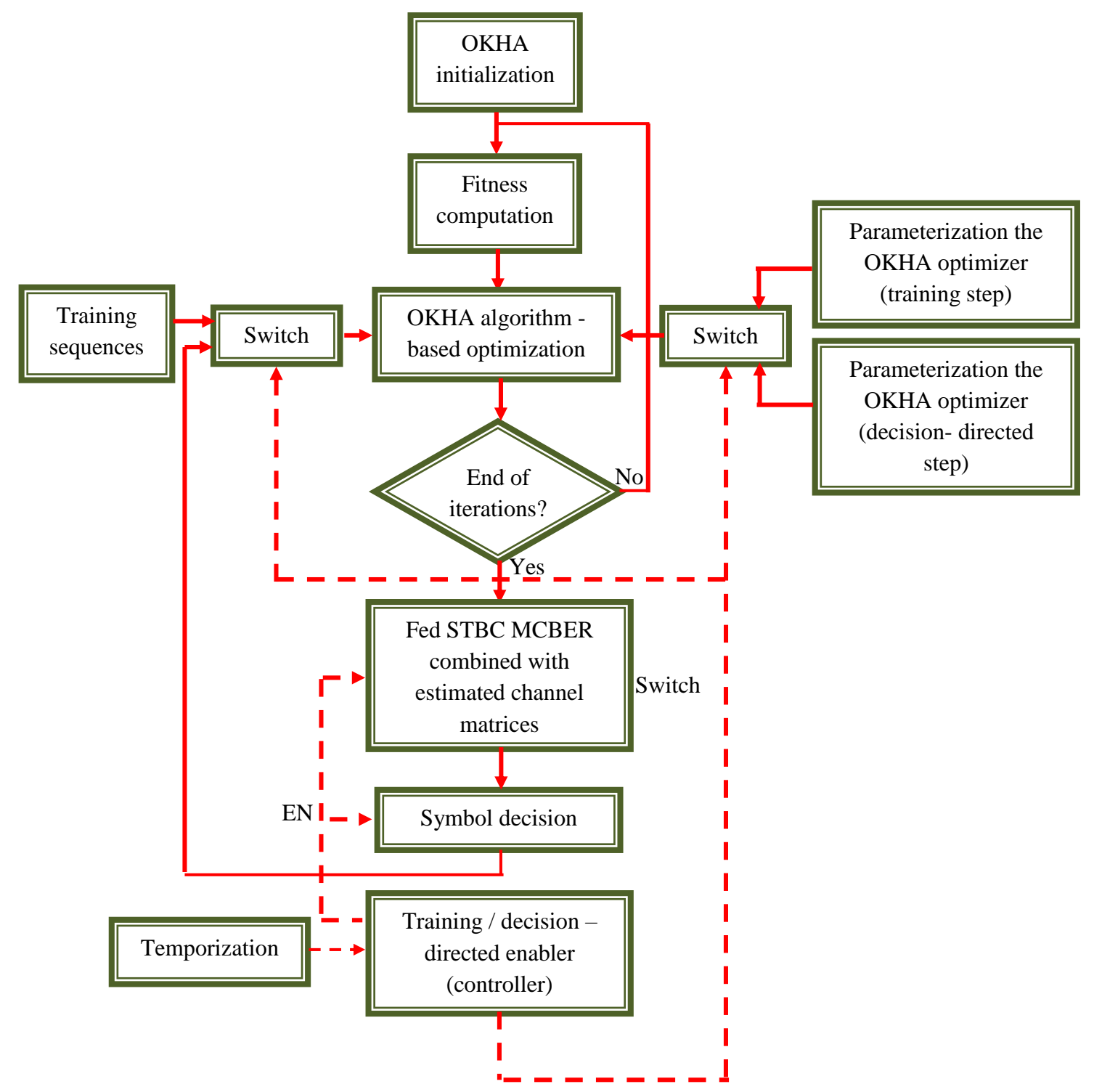

Figure.2 Flowchart of the OKHA-assisted channel estimation algorithm for STBC MC-CDMA

\section{Result and Discussion}

We have offered the results of our suggested methodology and have examined their presentation in this section. The proposed channel estimation in CDMA system is implemented in the MATLAB program, which was simulated in system specifications of 4 GB RAM and $3.2 \mathrm{GHz}$ Intel i-5 processor. The evaluation metrics employed are SER (Symbol Error Rate) and BER (Bit Error Rate).The performances of the proposed channel estimation are evaluated by means of intensive simulation trials in a Rayleigh fading channel fixing the following parameters: Number of subcarriers is 8 , transmission data rate is $1024 \mathrm{~Kb} / \mathrm{s}$, coherence bandwidth of the channel 2.1 MHz, Doppler spread of the channel $100 \mathrm{~Hz}$.

\subsection{Evaluation Metrics}

The evaluation of proposed MIMO MC-CDMA for STBC communication system using OKHA based optimal channel estimation technique is carried out using the following metrics.

Bit Error Rate: Bit Error Rate (BER) is defined as the rate at which errors occur in a transmission system. BER is the ratio of error bits received to the total bits sent.

Symbol Error Rate: Symbol Error Rate (SER) is defined as a number erroneously decoded information symbols (constellation points) divided by a total number of transmitted symbols.

Spectral Efficiency: Spectrum efficiency refers to the information rate that can be transmitted over a given bandwidth in a specific communication system. 


\subsection{Performance Analysis}

The main objective of proposed methodology is optimal channel estimation in MC-CDMA system. To improve the efficiency of the system in our work we choose the optimal channel using oppositional krill herd algorithm (OKHA). To encoding the transmitting signals, here we used Alamouti's spacetime block coding. In this section, we compare our proposed work with different approaches such as KHA based MC-CDMA system, without optimization based MC-CDMA system and Leandro et al. [16]. In [16], the author used Genetic Algorithm for channel estimation. But using GA, the author cannot find the optimal solution because using GA No guarantee of finding global maxima and its takes the maximum time for convergence. Using without optimization algorithm, we cannot obtain the minimum BER. To overcome the difficulty present in the KHA, we include the Oppositional based learning algorithm to the KHA for select channel matrix. To evaluate the proposed work, in this paper we used different configuration antenna sizes. Here, we carried out three types of result such as (i) $T x=2$, $R x=1$, user 2, subcarrier $S x=8$ (ii) $T x=2, R x=1$, user 4, subcarrier $S x=8$ (iii) $T x=2, R x=1$, user $6, S x=8$.

\section{* Case 1: $T x=2, R x=1, S x=8$ and user 2}

In this section, we explain the result obtained from proposed multi-user STBC MC-CDMA transmission System Based on Minimum Conditional BER Criterion and Oppositional krill herd algorithm (OKHA) assisted channel estimation using $T x=2$, $R x=1, S x=8$ and user 2 .

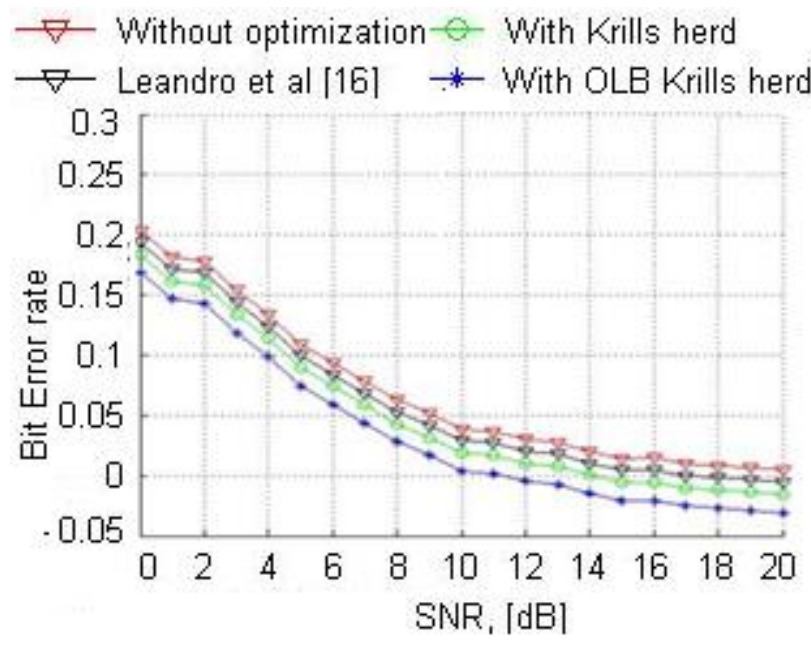

Figure.3 BER vs. SNR plots of MC-CDMA system for $T x=2, R x=1, S x=8$ and user 2

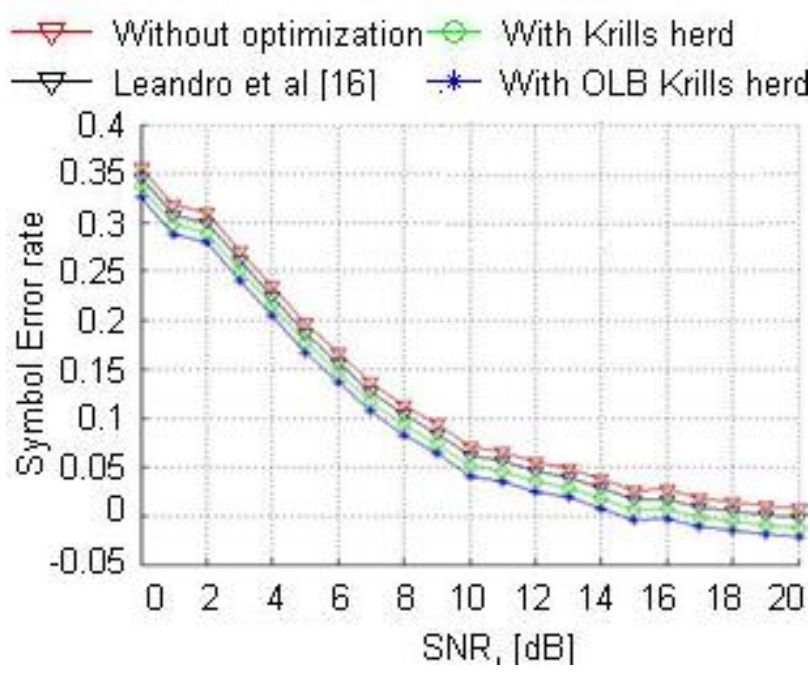

Figure.4 SER vs. SNR plots of MC-CDMA system for $T x=2, R x=1, S x=8$ and user 2

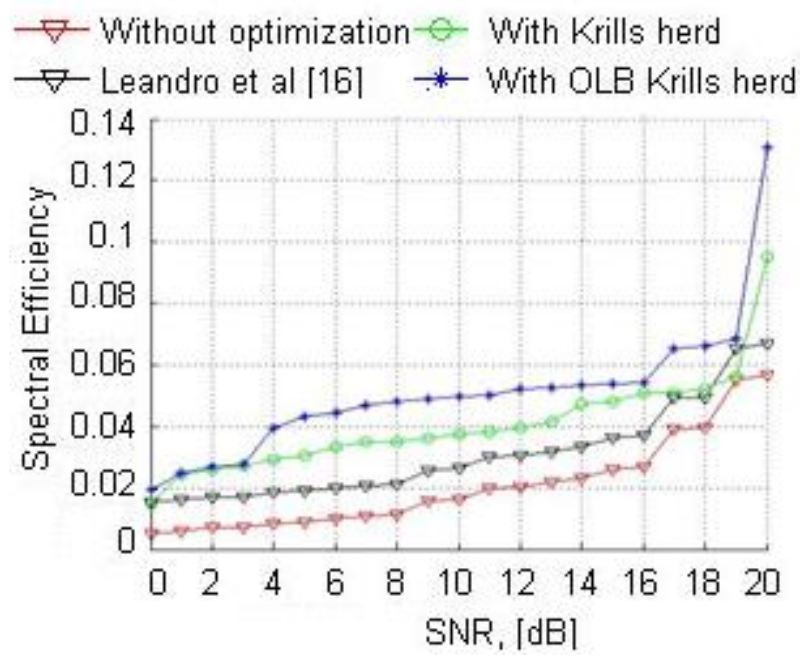

Figure.5 SNR vs. spectral efficiency of MCCDMA for $T x=2, R x=1, S x=8$ and user 2

The figure 3-5 shows the performance of the proposed methodology using different measures. The configuration of the system has two transmitting antennas, one receiver antenna, and two users. In this figure 1, Bit error Probability versus SNR dB, yield by the without optimization based MCBER detection, with krill herd algorithm based MCBER detection, Leandro et al. [16] and with OLB krill herd algorithm based MCBER detection. The optimization algorithm is used to optimize the channel matrix. The optimal channels value is used to reduce the error of the received signals. Here, The SNR varies from 0 to 20. The $\mathrm{x}$-axis represents the SNR value and y-axis represents the bit error rate. In figure 1 , we can see the proposed OKHA-based MCBER detection and without oppositional KHA based MCBER detection approach and Leandro et al. [16] clearly outperform without optimization based MCBER detection. 
Moreover, the proposed MCBER detection approach is slightly better than the KHA based MCBER detection and Leandro et al. [16] method. In figure 3, OKHA minimizes the bit error rate compare to other approach and the bit error rate is lower than 0.2. Moreover, figure 4 shows the performance of symbol error rate. The good transmitting system has the minimum SER value. From the figure 4, we understand our proposed OKHA minimize the bit error and the bit error rate is lower than 0.35 in figures 4. Similarly, figure 5 shows the performance of proposed work using spectral efficiency. Spectral efficiency refers to the information rate that can be transmitted over a given bandwidth in a specific communication system. It is a measure of how efficiently a limited frequency spectrum is utilized by the physical layer protocol, and sometimes by the media access control. Figure 5, we obtain the maximum spectral efficiency compare to other two approaches.

\section{* Case 2: $T x=2, R x=1$ and user 4}

In this section, we explain the result obtained from proposed multi-user STBC MC-CDMA transmission System Based on Minimum Conditional BER Criterion and Oppositional krill herd algorithm (OKHA) assisted channel estimation using $T x=2$, $R x=1, S x=8$ and user 4 . The effectiveness of the OKHA assisted channel estimation applied in such as MCBER framework which is shows from figures 68 . Here, used four transmitting antenna, two receiver antenna, and three users.

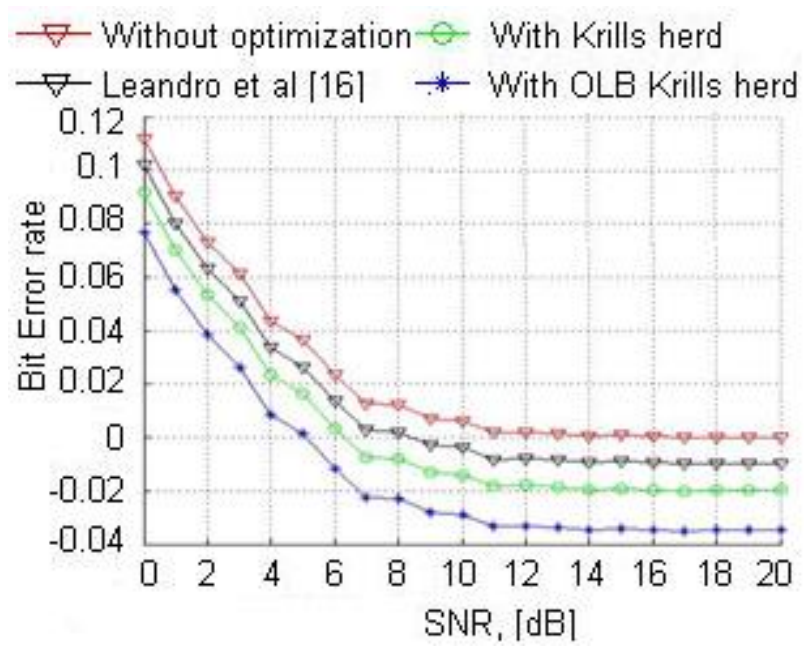

Figure.6 BER vs. SNR plots of MC-CDMA system for $T x=2, R x=1, S x=8$ and user 4

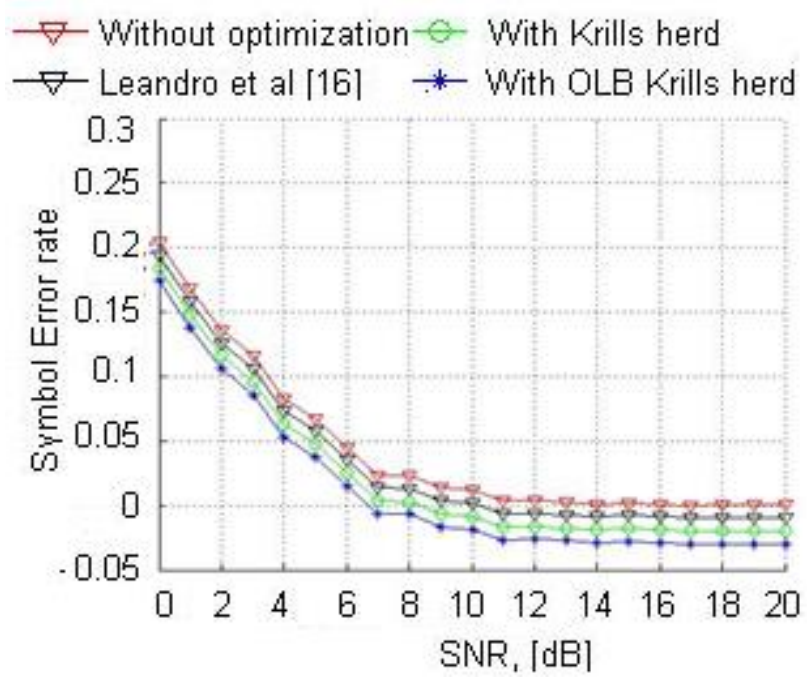

Figure.7 SER vs. SNR plots of MC-CDMA system for $T x=2, R x=1, S x=8$ and user 4

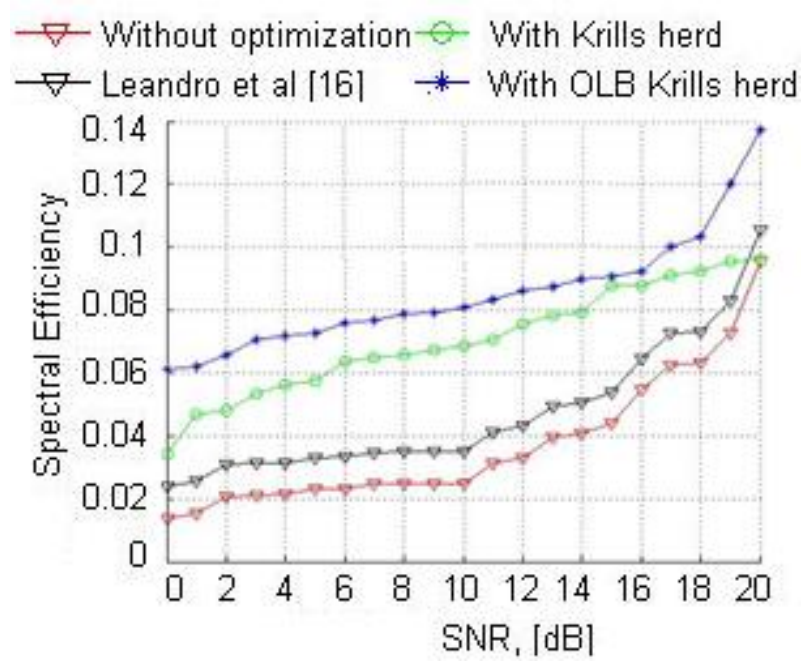

Figure.8 SNR vs. spectral efficiency of MCCDMA for $T x=2, R x=1, S x=8$ and user 4

Figure 6, shows the BER vs. SNR plots of MCCDMA system for $T x=2, R x=1$ and user 4 . Here, we obtain the BER of 0.075 . Moreover, figure 7 shows the SER vs. SNR plots of MC-CDMA system for $T x=2, R x=1, S x=8$ and user 4. Here, the SNR, [dB] varies from 0 to 20 . In this work, we compare our proposed OKHA based MCBER detection with KHA based MCBER detection, Leandro et al. [16] and without optimization based MCBER detection. Here, we obtain the symbol error rate is lower than 0.2 thus we can consider the OKHA minimize the SER. Moreover, figure 8 shows the performance of spectral efficiency versus SNR. Here, the proposed OKHA based MCBER detection method and KHA based MCBER detection approaches are got better result compare to the without optimization approach. The oppositional algorithm is used to increase the search space and increase the efficiency of the system. From 
the figure, we understand our proposed approach obtain the better result compare to the other methods.

\section{Case 3: $T x=2, R x=1$ and user 6}

In this section, we show the results obtained from the proposed methodology. Here, use the configuration of $T x=2, R x=1, S x=8$ and user 6 .

The below figure 9 shows the performance of the proposed system using BER vs. SNR plots. The $\mathrm{x}-$ axis represents the $S N R d B$ and $y$-axis represents the BER. The SNR value varies from 0-20. One can note that such an SNR, $[\mathrm{dB}]$, is decreasing with bit error rate and exhibits satisfactory values (i.e., lower than $0.04)$.

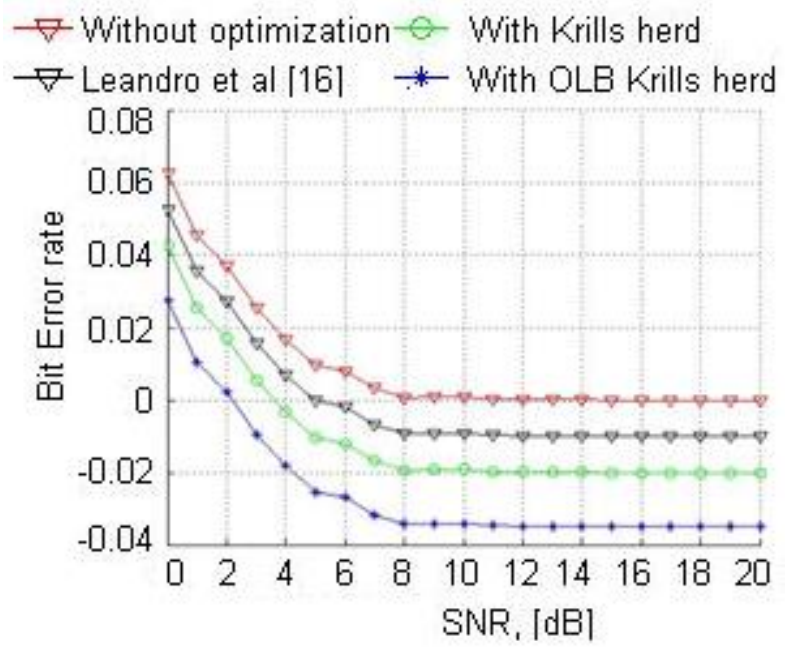

Figure.9 BER vs. SNR plots of MC-CDMA system for $T x=2, R x=1$ and user 6

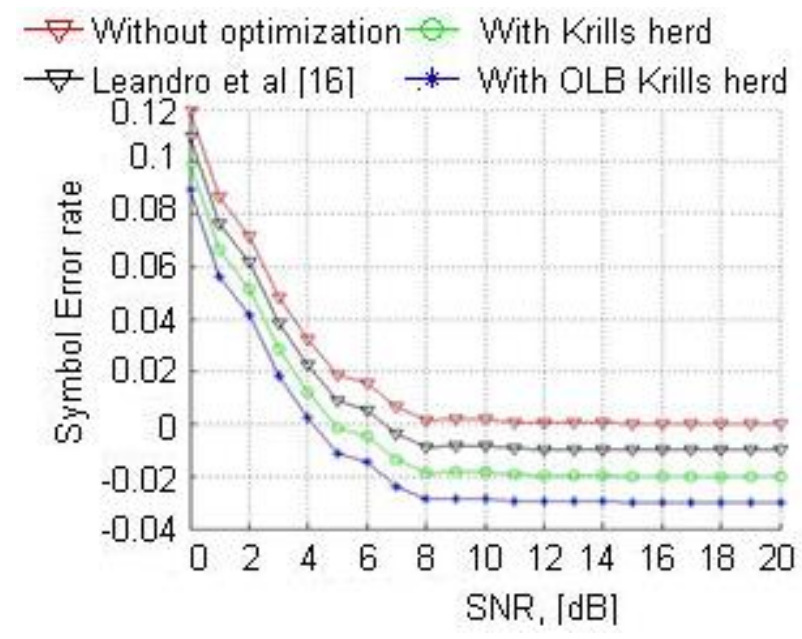

Figure.10 SER vs. SNR plots of MC-CDMA system for $T x=2, R x=1$ and user 6

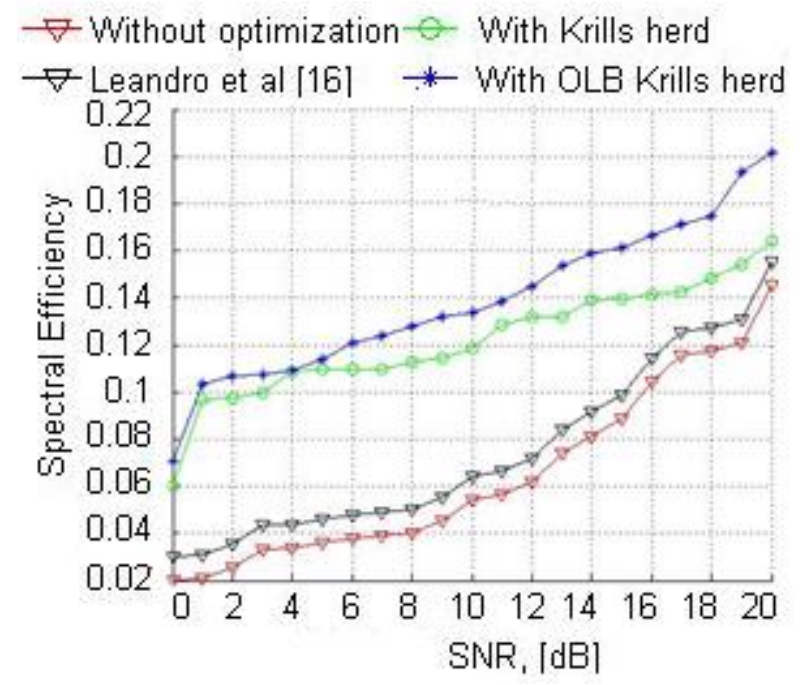

Figure.11 SNR vs. spectral efficiency of MCCDMA for $T x=2, R x=1$ and user 6

Figure 10 shows the performance of the proposed approach using SER vs.SNR. From the figure 10, one can see that the symbol error rates of all the systems decrease. Observe from this figure that symbol error rate was reduced and lower than 0.1 . The figure 11 shows that spectral efficiency versus SNR, and yield without optimization, with KH, Leandro et al. [16] and with OKHA. The SNR is varying from 0 to 20 . Using without optimization we obtain the spectral efficiency of 0.12, using KHA we obtain the spectral efficiency vary up to 0.16 , Leandro et al. [16] method achieve 0.15 and our proposed approach achieves 0.2 . From the result, we clearly, understand our proposed approach achieves the better result compare to other approaches.

\section{Conclusion}

In this paper, we introduced and discussed a Performance of MIMO MC-CDMA for STBC communication System using OKHA based optimal channel estimation. Here, we properly explained the channel estimation calculation using OKHA. Further on, the achieved estimated channel matrices have been employed to implement the canonical MMSE Multi-User Detection. Simulation comparisons are conducted with the proposed algorithm, the Krill herd algorithm and without optimization approach respectively. The system is evaluated based on different configurations. The results show that the proposed oppositional krill herd algorithm is more efficient in terms of BER, SER, and Spectral efficiency. In future, we will increase the number of antennas at both transmitter and receiver without introducing any interference in between the antennas. 


\section{References}

[1] R. Abdallah and M. Mokhtar, "Performance enhancement of asynchronous MC-CDMA system with carrier frequency offset using successive interference cancellation”, Personal, Indoor and Mobile Radio Communications, Vol. 3, 2003.

[2] M. H. Shoreh, A. Fallahpour and J. A. Salehi, "Design concepts and performance analysis of multicarrier CDMA for indoor visible light communications", Journal of Optical Communications and Networking, Vol. 7, No. 6, pp. 554-562, 2015.

[3] L. Hanzo, M. Münster, B. J. Choi and T. Keller, "OFDM and MC-CDMA for Broadcasting Multi-User Communications", WLANs and Broadcasting, Wiley, 2003.

[4]K. Fazel, "On the performance of convolutionallycoded CDMA/OFDM for mobile communication system", Proc. IEEE PIMRC'93, pp. 468-472, 1993.

[5]Y. Nathan, J. P. Linnartz and G. Fettweis "Multicarrier CDMA in indoor wireless radio networks", Proc. IEEE PIMRC'93, pp. 109-113, 1993

[6] R. Prasad and S. Hara, "Overview of multi-carrier CDMA", IEEE Commun. Mag, Vol. 35, No. 12, pp. 126-133, 1997.

[7] A. Phasouliotis and K. C. Daniel, "Layered spacetime receiver for downlink multiple-input multiple-output multi-carrier code division multiple access systems", IET communications, Vol. 5, No. 13, pp. 1907-1917, 2011

[8]K. Rasadurai, P. S. Kumar and N. Kumaratharan, "Performance enhancement of MC-CDMA system using LDPC codes", Information \& Communication Technologies, pp. 78-62, 2013.

[9]N. Figueiredo, L. P. Linde and J. H. VanWyk, "Performance of a transmit diversity MIMO MCCDMA communication system employing cyclically rotated complete complementary codes", In AFRICON, IEEE, pp. 1-5, 2013.

[10]S. Won and L. Hanzo, "Initial synchronisation of wideband and UWB direct sequence systems:
Single-and multiple-antenna aided solutions", IEEE Communications Surveys \& Tutorials, Vol. 14, No. 1, pp. 87-108, 2012.

[11]K. Pinhui and Z. Zhou. "A Generic Construction of Z-Periodic Complementary Sequence Sets with Flexible Flock Size and Zero Correlation Zone Length", IEEE Signal Processing Letters, Vol. 22, No. 9, pp. 14621466, 2015.

[12]S. Han, R. Venkatesan, H. H. Chen, W. Meng, C. $\mathrm{Li}$ and P. Y. Yang, "A complete complementary coded MIMO system and its performance in multipath channels", IEEE Wireless Communications Letters, Vol. 3, No. 2 pp. 181184, 2014.

[13]Z. Liu, Y. L. Guan and H. H. Chen, "FractionalDelay-Resilient Receiver Design for Interference-Free MC-CDMA Communications Based on Complete Complementary Codes", IEEE Transactions on Wireless Communications, Vol. 14, No. 3, pp. 1226-1236, 2015.

[14]T. Sälzer and D. Mottier, "Transmit beam forming for SDMA in multicarrier CDMA downlink of a per subcarrier basis," In Proc. IEEE Int. Conf. Telecommun. Vol. 1, pp. 793-798, 2003.

[15]S. Alamouti, "A simple transmit diversity technique for wireless communications", IEEE J. Sel. Areas Commun, Vol. 16, No. 8, pp. 14511458, 1998.

[16] L. D’Orazio, C. Sacchi, M. Donelli, J. Louveaux and L. Vandendorpe, "A Near-Optimum Multiuser Receiver for STBC MC-CDMA Systems Based on Minimum Conditional BER Criterion and Genetic Algorithm-Assisted Channel Estimation", EURASIP Journal on Wireless Communications and Networking, No. 1, pp. 351494, 2011

[17] J. Yang, Y. Sun, J.M. Senior, and N. Pem, "Channel Estimation for Wireless Communications using Space-Time Block Coding Techniques", IEEE International symposium on Circuits and Systems. Vol. II, pp. 220-223, 2003. 